\title{
TIME EVOLUTION OF RELEASED HOLE ARRAYS INTO MEMBRANES VIA VACUUM SILICON MIGRATION
}

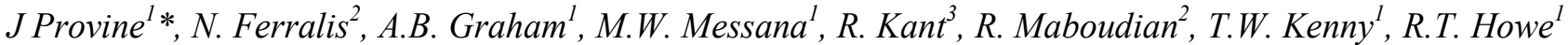 \\ ${ }^{1}$ Stanford University, Stanford, California, USA ${ }^{2}$ University of California, Berkeley, California, USA \\ ${ }^{3}$ University of California, San Francisco, California, USA
}

\begin{abstract}
This work reports on experimental evaluation of the evolution of suspended silicon hole arrays into membranes through silicon migration in an ultrahigh vacuum (UHV) environment. Multiple identical hole arrays were imaged and measured through scanning electron microscopy after different migration conditions. Many designs of hole arrays eventually progress to continuous membranes, however, despite imperceptible differences in initial geometry the intermediate steps indicate significant non-uniformity of migration. Small deviations from complete volume conservation were observed and evidence of silicon evaporation from the surface supported this volumetric change.
\end{abstract}

\section{INTRODUCTION}

As microfabrication continues to push toward smaller and smaller dimensions, surface effects become more dominant. Cost of equipment is a limiting factor for true nanostructuring based on top-down fabrication alone. To this end, silicon migration is an attractive technique to add to the micromachining tool box because of its capability to produce three dimensional, smooth structures resulting from a surface curvature minimization method.

Silicon migration has been utilized to smooth rough surfaces [1], modify optical components [2], and create nanofluidic devices [3]. More recently, efforts on analyzing migrated silicon for controlled time steps indicate different gaseous environments affect the migration process [4]. Previous studies of silicon migration have not reported intermediate monitoring of the process, instead allowing migration to approach an asymptotic equilibrium structure. For instance, the migration of silicon trenches to form continuous membranes was reported [5].

Much of the literature on silicon migration has focused on migrating a given top-down, lithographically defined feature into its final, fully curvature minimized structure. However, to fully realize the potential of silicon migration as a tunable fabrication technique, it is important to understand the progression of a structure toward the lowest curvature state via silicon migration so we can utilize the intermediate structures as well. In an attempt to study this regime, we created a large sample set of similar structures to perform a range of comparative migration experiments. We chose released uniform hole arrays as our test structure because of the ability to study migration phenomena at both large (100s of microns) and small scales (less than 1 micron) while simultaneously observing millions of identical "unit cells" undergoing identical migration treatments.

\section{EXPERIMENTAL SETUP}

\section{Test Structure Preparation}

Starting with an SOI with a $4 \mu \mathrm{m}$ thick, $<100>$ oriented device layer, hole arrays of various dimensions were fabricated through optical lithography and RIE. A thermally grown $\mathrm{SiO}_{2}$ hard mask was grown, and patterned using an ASML wafer stepper to expose the single mask layer. The oxide layer was reactively ion etched in $\mathrm{Cl}_{2}$ chemistry, then the same masking layer was used to reactively ion etch the device layer silicon with a combination of $\mathrm{HBr}$ and $\mathrm{Cl}_{2}$. The device layer of the SOI was released by vapor $\mathrm{HF}$ etch of the buried oxide layer, which also served to remove the remaining oxide hard mask. Figure 2 shows an SEM of a representative asfabricated device.

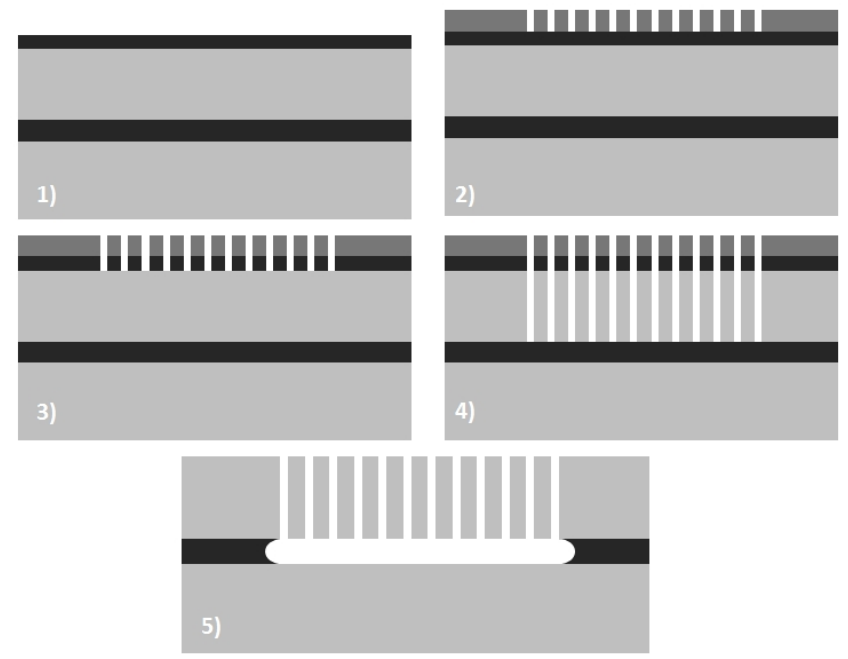

Figure 1: Schematic of the process flow sample preparation. 1) Growth of thermal oxide hard mask. 2) Photolithographic patterning of hole array. 3) Pattern transfer into oxide hard mask by RIE. 4) Pattern transfer into device layer Si by RIE. 5) Device release by vapor $H F$.

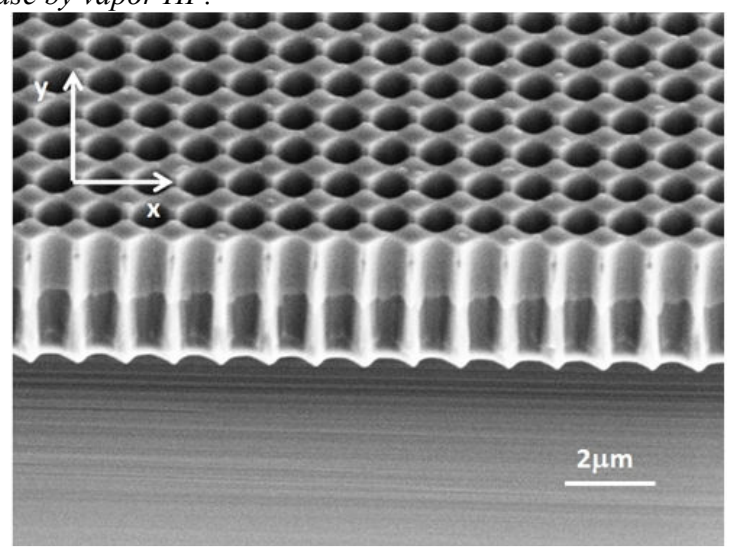

Figure 2: Scanning electron micrograph of a fabricated perforated membrane with no Si migration that has been cleaved through the membrane. The sample is tilted $45^{\circ}$ with respect to the imaging beam. For this sample the pitch is $1100 \mathrm{~nm}$ and the diameter of the holes is $900 \mathrm{~nm}$. Note the slight asymmetry in $x$ and $y$ directions.

Silicon migration was performed at various temperatures and times in UHV $\left(10^{-9}\right.$ Torr). Samples are heated by electron bombardment on the back of the sample, using a tungsten filament. Temperature is monitored using a K-type thermocouple that is placed in close proximity to the sample holder. The thermocouple is calibrated using comparative measurements performed with an optical pyrometer on a molybdenum sample. The statistical error in temperature measurements is $5^{\circ} \mathrm{C}$. We report $20^{\circ} \mathrm{C}$ as the overall uncertainty to account for potential heater drift after several 
heating cycles. The samples were cleaved through the hole arrays and imaged using a SEM to track the progress of silicon migration.

\section{RESULTS \& DISCUSSION}

\section{Time Evolution at a Constant Temperature}

Figures 3 \& 4 show the time evolution under vacuum migration of a particular hole array at an imaging angle of $45^{\circ}$ and $90^{\circ}$, respectively. Because of a lithography, issue the hole array is initially non-symmetric in the $\mathrm{x} \& \mathrm{y}$ directions with many sharp features. This non-uniformity manifests within a few minutes of migration as the formation of ridges in the x-direction as the hole array begins to seal across the surface where the buried oxide has been removed. Initially these sharp lithographically defined features and the sharp edges from the etch hard mask are removed to produce the overall smaller curvature of the ridge system. After 16 minutes the membrane is continuous and the ridges continue to migrate into a smooth membrane. By 64 minutes the membrane has some noticeable features left from the original hole array but is otherwise an uninterrupted silicon membrane over the entire array area. The system asymptotically approaches a smooth flat membrane and the difference between 32 and 64 minutes of anneal time is small, as fine features begin to be removed.

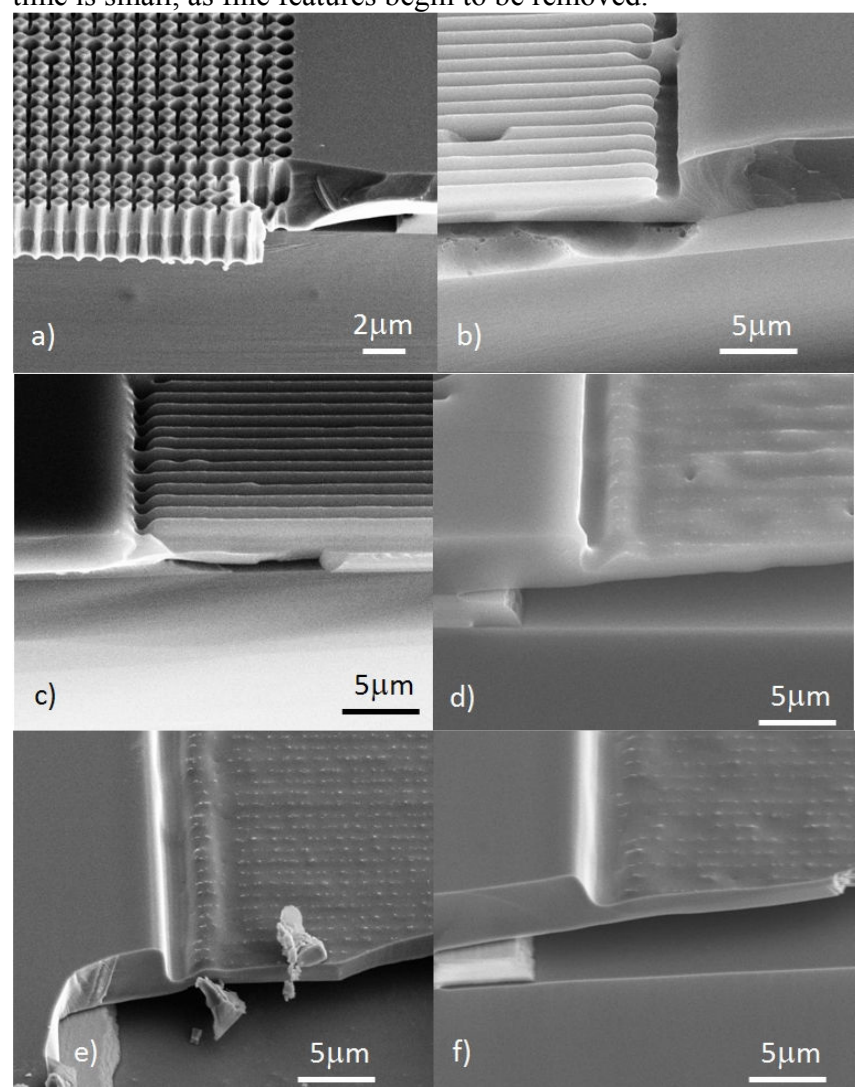

Figure 3: SEMs of a hole array with pitch of 1000nm and hole diameter of $800 \mathrm{~nm}$. The sample is a $45^{\circ}$ angle to the imaging beam. Migration performed at $1100^{\circ} \mathrm{C}$ for: a) No migration; b) 4 minutes; c) 8 minutes; d) 16 minutes; e) 32 minutes; and f) 64 minutes.

A close examination of the sample without any anneal performed indicates that the sidewalls from the $\mathrm{Si}$ etch are not perfectly straight and thus the holes are slightly wider at the top than at the bottom. This asymmetry is a contributing factor to the membranes sealing from the bottom up. In Figure 4 the changing thickness of the membrane can be clearly observed. As the perforated membrane migrates to a continuous membrane, we can monitor the relative thickness in comparison to the relatively unchanging thickness of the unpatterned region of the device layer.
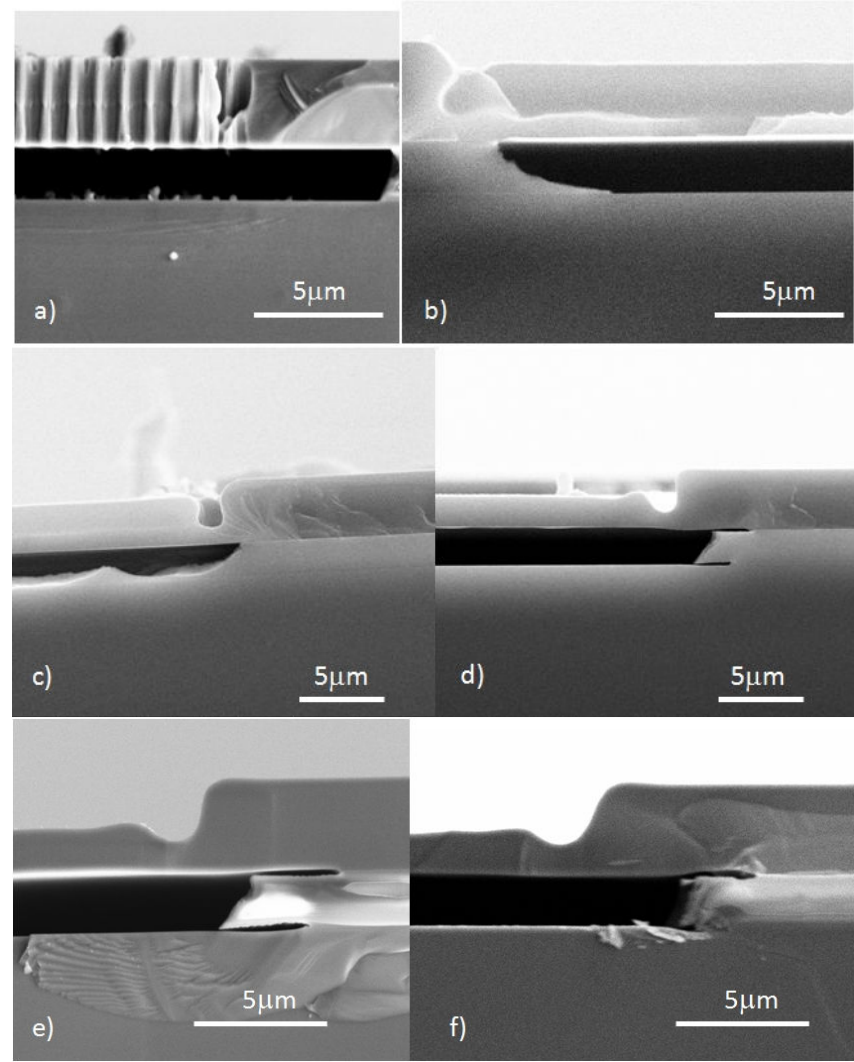

Figure 4: SEMs of a hole array with pitch of 1000nm and hole diameter of $800 \mathrm{~nm}$. The sample is a $90^{\circ}$ angle to the imaging beam. Migration performed at $1100^{\circ} \mathrm{C}$ for: a) No migration; b) 4 minutes; c) 8 minutes; d) 16 minutes; e) 32 minutes; and f) 64 minutes.

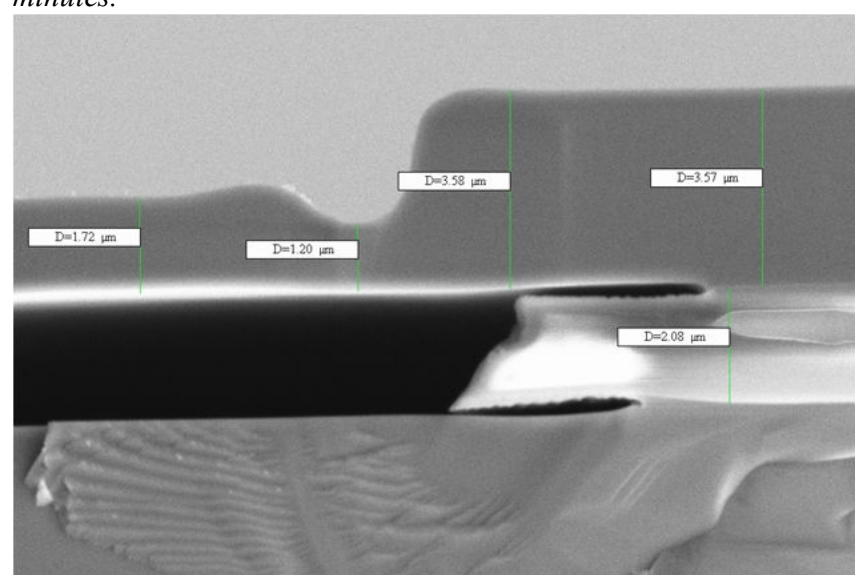

Figure 5: SEM with measurements of various dimensions for a sample after 64 minutes of vacuum anneal.

In Figure 5, measurements of various dimensions of continuous membrane formed after 64 minutes of vacuum anneal at $1100 \mathrm{C}$ are given. For a hole array with $1000 \mathrm{~nm}$ pitch and $800 \mathrm{~nm}$ diameter, the etched volume is $400^{2} \pi / 1000^{2} \approx 0.50$. The thickness of the membrane is measured as $1.72 \mu \mathrm{m}$, which can be compared to the original device layer thickness (after oxide hard 
mask growth) of $3.57 \mu \mathrm{m}$ for a final thickness that is $\sim 0.48$ of the original thickness. This is similar but slightly smaller than the amount expected if volume is conserved in the migration.

\section{Silicon Evaporation}

The concern about volume conservation during this process stems from previous work [6] and the authors experience with silicon evaporation at these temperatures and pressures. The evidence of evaporation is confirmed by SEM of regions separate from the hole array where pits of removed silicon are observed (Figure 6). These pits are not present in the initial wafers and present themselves as an overall "hazy" appearance after annealing at higher temperatures, in this case $1100^{\circ} \mathrm{C}$. At these elevated temperatures and reduced pressures there are two competing surface modification processes: 1) volume conserving silicon migration and 2) volume non-conserving silicon evaporation. Some of the evaporated silicon will be redeposited on the sample substrate, but the majority will be deposited on the annealing chamber. The balance of these two competing processes must be understood for manipulation of surfaces at the nanoscale. The rate of migration is lower for a lower temperature, but the effect of evaporation is also reduced as can be seen in Figure 7 where an anneal of 64 minutes in $1000^{\circ} \mathrm{C}$ is performed.

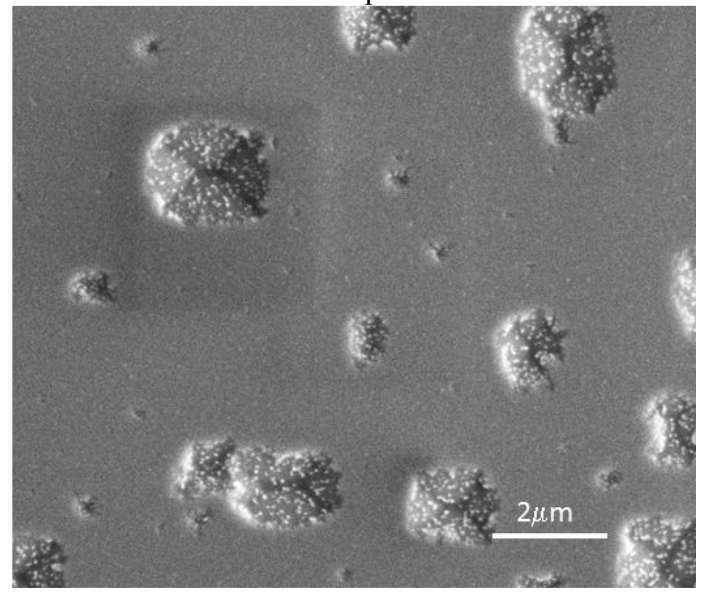

Figure 6: SEM of a region of a sample distant from a hole array showing pits formed by silicon evaporation sites. This anneal was performed at $1100^{\circ} \mathrm{C}$ for 64 minutes and can be compared to the lower temperature anneal in Figure 7.

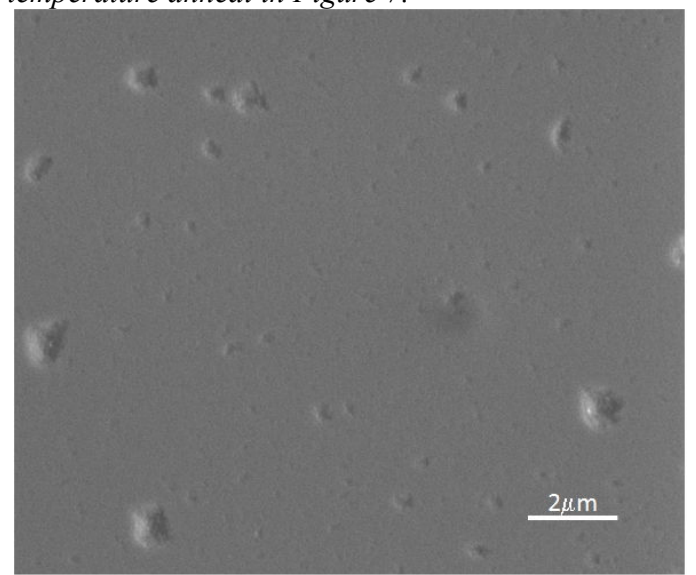

Figure 7: SEM of a region of a sample distant from a hole array showing pits formed by silicon evaporation sites. This anneal was performed at $1000{ }^{\circ} \mathrm{C}$ for 64 minutes and can be compared to the higher temperature anneal in Figure 6.

\section{Non-uniform Migration}

Silicon migration is thought of as an energy minimization process, and as such it has been considered as a method for nanostructuring on the large lateral scale of a chip or even wafer. When pushing structures to their asymptotic final state, this is a good supposition to employ. However, during the process of evolution through intermediate steps toward the final structure, migration between nearly identical original shapes can lead to drastically different structures. Examples of this phenomenon can be seen in Figures 8, 9, and 10.

In Figure 8, a large area where the migration rate has been slower than in the surrounding structure is shown. This is for an anneal of 96 minutes at $1100^{\circ} \mathrm{C}$ for a hole array with pitch of $1200 \mathrm{~nm}$ and diameter of $1000 \mathrm{~nm}$. This type of non-uniformity was seen repeatedly in the hole arrays with larger feature sizes. One possible reason for this behavior is non-uniform heat conduction as the these regions of lower migration rate are always at the center of the membranes where vacuum gap resulting from the released buried oxide and as opposed to the continuous solid material at the boundaries of the membranes. Also in Figure 8, there are local migration defects that can be seen. While significant care was taken with the cleanliness of these samples it is possible these defects arise from particulate contamination or as a possible by-product of silicon evaporation on the sample.

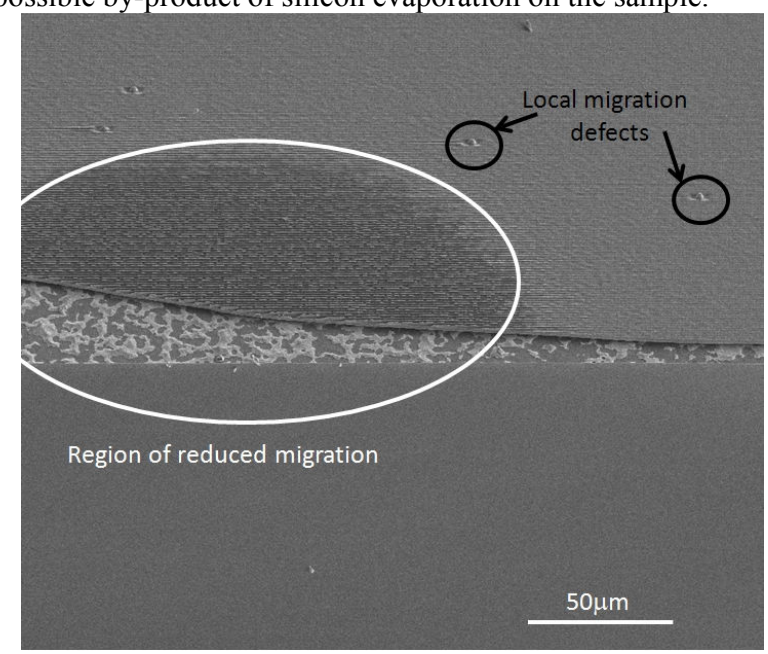

Figure 8: SEM showing a hole array with initial condition of $1200 \mathrm{~nm}$ pitch and $1000 \mathrm{~nm}$ diameter after 96 minutes of vacuum anneal at $1100^{\circ} \mathrm{C}$. Both a large region of reduced migration rate at the center of the membrane and some localized migration defects are highlighted.

Figure 9 indicates another type of consistently observed nonuniformity in migration, namely incomplete membrane formation. A hole array with initial lithographically defined dimensions of a pitch of $800 \mathrm{~nm}$ and diameter $600 \mathrm{~nm}$ was not sufficiently etched to reached the buried oxide. After a vacuum anneal of $1000^{\circ} \mathrm{C}$ for 96 minutes the device layer silicon has migrated into a double layer (Figure 9a) over much of the lateral dimension of the hole array. But only a few microns away from the region of Figure 9a, this migration process is still ongoing and regions where the two silicon layers are still connected is observed (Figure 9b). Further, the number of "unit cells" joined in the migration step is nonuniform even in consecutive cells. 


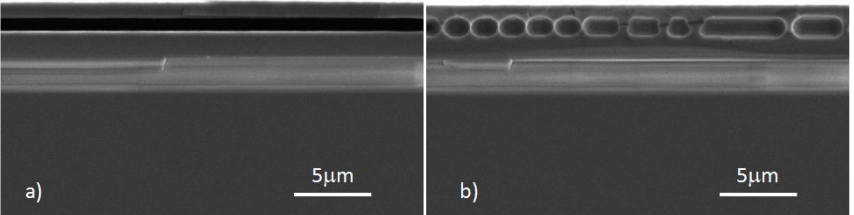

Figure 9: SEM of two neighboring regions in a membrane formed from a hole array of initial dimensions $800 \mathrm{~nm}$ pitch and $600 \mathrm{~nm}$ diameter. The anneal conditions were $1000{ }^{\circ} \mathrm{C}$ for 96 minutes. The double silicon layer is caused by an etch that did not penetrate to the buried oxide. The formation of the double silicon layer can be seen to be different in even neighboring cells.

Finally, Figure 10 reveals a striking case of non-uniformity that could be thought of as the combination of the phenomena shown in Figures 8 and 9. Upon observation of the original sample (Figure 10a) the hole array appears to be comprised of many identical unit cells. However, migration under different temperature and time steps produce a collection of shapes formed from highly non-uniform migration rates for neighboring unit cells. It should be noted that the mechanism for such non-uniformity is not fully understood currently. However, it is clear that in systems without perceptible non-uniformity at a given imaging scale, massive non-uniformity can arise during silicon migration.

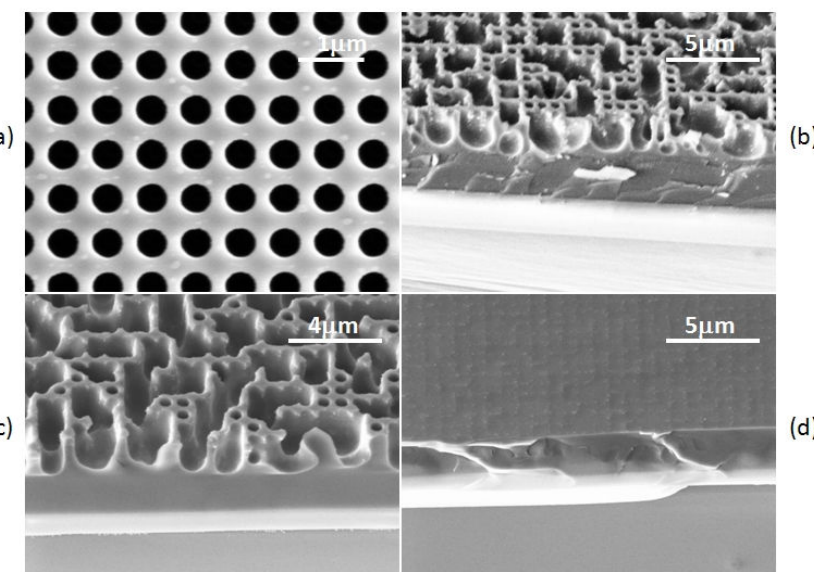

Figure 10: SEMs of a hole array with $700 \mathrm{~nm}$ pitch and $500 \mathrm{~nm}$ diameter at various stages of migration. a) no migration, b) 32 minutes at 1100C, c) 64 minutes at 1000C, and d) 64 minutes at 1100C. At intermediate steps the progression of the migration is highly non-uniform laterally, but the final membrane is fully formed and continuous.

\section{CONCLUSION \& FUTURE WORK}

Through the production of a large sample set of similar structures, we were able to undertake a systematic evaluation of silicon migration on the micron scale. All of the hole array designs eventually migrated to form continuous membranes of solid silicon. The time evolution of the migration was observed through electron microscopy. The membranes were observed to seal first at the bottom and approach an asymptotic, smooth membrane by first reducing sharp local features that larger scale curved features.

We measured small deviations in volume of the original hole array and the final membrane. This change in volume is on the order of $2 \%$ and may fall within experimental error. An additional factor indicating non-conservation of device layer volume is the observed nanostructuring from silicon surface evaporation.

Additionally, highly non-uniform migration behavior was observed on both a large (several 10s of microns) and small scale (less than 1 micron). The large scale difference could be attributed to deviations in temperature due to thermal conductivity. The cause of the small scale non-uniformity is more difficult to account for as systems that appear uniform at a certain imaging scale become massively non-uniform at that same scale during migration, with even neighboring unit cells deviating significantly.

Further work to understand the interaction between migration and evaporation at these pressure and temperatures will be essential to determining the long-term usefulness of silicon migration as a tunable fabrication technique. In addition, we have begun to perform surface analysis of the migrated samples to determine various material properties after migration such as crystallinity, film stress, and material composition.

\section{ACKNOWLEDGEMENTS}

This work was supported by DARPA MTO Center on Interfacial Engineering in Microelectromechanical Systems (CIEMS) (HR 0011-06-1-0049; Dr. Dennis Polla, Program Manager)

\section{REFERENCES}

1. T. Sato, et al, Micro-structure Transformation of Silicon: A Newly Developed Transformation Technology for Patterning Silicon Surfaces using the Surface Migration of Silicon Atoms by Hydrogen Annealing, Jpn. J. Appl. Phys. Vol 39 (2000), pp 5033-5038.

2. S. Kim, et al, "Interface Quality Control of Monolithic Photonic Crystals by Hydrogen Annealing," Conference on Lasers and Electro-Optics (CLEO) 2008, San Jose, CA.

3. T. A. Zangle, et al, "Microfluidic device with integrated nanopores for protein detection," to be presented at the $13^{\text {th }}$ Int. Conf. on Miniaturized Systems for Chemistry and Life Sciences (mTAS 2009), Jeju, Korea, Nov. 1-5, 2009.

4. R. Kant, et al, "Micro-scale silicon migration in high temperature, low pressure, inert ambient", Electrochemical and Solid-State Letters 12 (2009), H437-H440.

5. R. Kant, et al, "Suspended microstructures made using silicon microstructures," $15^{\text {th }}$ Int. Conf. on Solid-State Sensors, Actuators and Microsystems, Denver, Colorado, June 21-25, 2009.

6. L. Zhong, et al, "Determination of Silicon Evaporation Rate at $1200^{\circ} \mathrm{C}$ in Hydrogen," Applied Physics Letter, 67 (26), December 1995.

\section{CONTACT}

*J Provine, tel: +1-650-644-04-3; jprovine@stanford.edu 